\title{
EDITORIAL
}

\section{New CMAJ and CMAJ Open policy permitting preprints}

\author{
Diane Kelsall MD MEd
}

Cite as: CMAJ 2019 July 8;191:E752. doi: 10.1503/cmaj.190780

$\mathbf{T}$ he launch of the latest preprint server, MEDRxiv, specifically targeted at the clinical research community, is a sign of growing interest in preprints among health researchers. Preprints are scholarly papers that are posted by authors in an openly accessible platform, usually before submission to a journal for formal publication. ${ }^{1}$ There are now about 45 preprint servers in operation across academic disciplines, and the number of articles being posted is growing rapidly. In response to this trend, we have developed a policy on preprints that permits papers deposited in a preprint server to be considered for publication in CMAJ and CMAJ Open.

For researchers, preprints facilitate early and rapid dissemination of their work among the research community, which provides an opportunity for them to receive feedback from a wide audience that may improve the draft paper before journal submission. Preprints may help authors to establish precedence for a research finding or find potential collaborators for future work. ${ }^{1,2}$

More broadly, preprints may help reduce research waste and publication bias. ${ }^{2}$ Even good studies may have difficulty getting published at times because of negative results or limited generalizability. Preprint servers can facilitate dissemination of these study findings among the research community. And researchers can check whether there is recent research in an area they are considering exploring, which could reduce the likelihood of duplicative studies.

There are inherent risks associated with posting preprints. ${ }^{1}$ While the goal of most medical research is to have the findings incorporated into clinical practice or used to develop health policy, draft papers posted on preprint servers have undergone neither peer review nor rigorous vetting and editing at a reputable journal. Application of the findings of non-peer-reviewed studies may cause harm. To address this concern, papers on preprint servers are now more clearly labelled as preliminary reports that have not undergone peer review. Some servers, most notably MEDRxiv, have gone further and implemented additional safeguards, including screening for competing interests, ethics approval and plagiarism. ${ }^{2}$

CMAJ and CMAJ Open will consider for publication papers that have previously been deposited in a preprint server and for which the authors retain copyright. Authors should inform us on submission if a preprint version exists and provide the link for the information of editors and reviewers. We encourage authors to consider revising their paper before submission in response to comments on the preprint.
On publication of a paper in CMAJ or in CMAJ Open, authors will be required to ensure a link is added on the preprint to the published paper, along with the full citation - and the journal will add a reciprocal link. However, neither revised versions of the manuscript made during the journal review process nor the published version should replace the draft version on the preprint site. To protect the public, authors should not seek any media coverage of the findings reported in their preprint, and if contacted by media outlets, authors should make it clear that the preprint version is a preliminary report that has not been peer reviewed.

What should authors look for in a preprint server? We suggest that authors consider using nonprofit, community-based and open access preprint servers that provide preliminary screening for expected standards of research integrity and have policies for handling preprints that violate those standards. ${ }^{1}$ Posted preprints should be marked clearly as preliminary, non-peer-reviewed work. To facilitate future publication, the author must retain copyright for the preprint. Additionally, the server should assign a unique digital object identifier (DOI) for each preprint to facilitate discovery and linkage to the final published version. Posting on preprint servers is typically free for authors at this time.

The rise of preprints parallels a general trend in the publishing world to share research findings quickly and efficiently. With the expansion of open access journals such as CMAJ Open, it has become easier than ever to publish methodologically sound negative studies or studies with limited generalizability. And many journals, including those published by the CMAJ Group, are working hard to substantially reduce the time to publication of research. This new policy on preprints will help CMAJ achieve its mission of championing knowledge that matters for the health of Canadians and the rest of the world.

\section{References}

1. COPE discussion document: preprints. Version 1. Hampshire (UK): Committee on Publication Ethics (COPE); March 2018. Available: https://publicationethics. org/files/u7140/COPE_Preprints_Mar18.pdf (accessed 2018 June 21).

2. Rawlinson C, Bloom T. New preprint server for medical research. BMJ 2019;365:12301.

Competing interests: See www.cmaj.ca/site/misc/cmaj_staff.xhtml

Affiliation: Editor-in-Chief (interim), CMAJ

Correspondence to: CMAJ editor, cmaj@cmajgroup.ca 